\title{
Robot System for Preparing Lymphocyte Chromosome
}

\author{
ISAMU HAYATA ${ }^{1)}$, HIROYOSHI TABUCHI ${ }^{2)}$, AKIRA FURUKAWA ${ }^{1)}$, \\ NOBUO OKABE ${ }^{3)}$, MIKIO YAMAMOTO ${ }^{1)}$, AND KOKI SATO ${ }^{1)}$ \\ ${ }^{1)}$ National Institute of Radiological Sciences, Anagawa 4-9-1, Chiba-shi 260, Japan \\ ${ }^{2)}$ Umetani Precison Co., Minamishinagawa 6-12-3, Shinagawa, Tokyo 140, Japan \\ ${ }^{3)}$ Research Laboratory, Nikon Co., Nishiohi 1-6-3, Shinagawa, \\ Tokyo 140, Japan
}

\begin{abstract}
Automated System/Radiation dosimetry/Chromosome preparation/culture/harvest
Towards the automatization of the scoring of chromosome aberrations in radiation dosimetry with the emphasis on the improvement of biological preparations, the conventional culture and harvesting method was modified. Based on this modified method, a culture and harvest robotic system (CHROSY) for preparing lymphocyte chromosome was developed. The targeted points of the modification are as in the following. 1. Starting culture with purified lymphocytes in a fixed cell number. 2. Avoiding the loss of cells in changing the liquids following centrifugalization. 3. Keeping the quantity of the liquids to be applied to the treatments of cells fixed. 4. Building a system even a beginner can handle. System features are as follows. 1. Operation system: Handling robot having 5 degrees of freedom; a rotator incubator with an automatic sliding door; units for setting and removing pipette tips; a centrifuge equipped with a position adjuster and an automatic sliding door; two aluminum block baths; two nozzles as pipettes and aspirators connected to air pumps; a capping unit with a nozzle for $\mathrm{CO}_{2}$ gas; a compressor; and an air manipulated syringe. 2. Control system: NEC PC-9801RX21 with CRT; and program written in Basic and Assembly languages on MS-DOS. It took this system 2 hours and 25 minutes to harvest 2 cultures. A fairly good chromosome slide was made from the sample harvested by CHROSY automatically.
\end{abstract}

\section{INTRODUCTION}

Chromosome aberrations caused in the peripheral blood lymphocytes serve as the best biological indicators of the exposure to the ionizing radiation. Advantages of applying the chromosome analysis method to biological dosimetry can be summarized as follows. 1) Chromosomes reflect extremely sensitive reaction, and aberrations increase in proportion to the radiation dose even at the level of an acute X-ray exposure of $50 \mathrm{mGy}^{1)}$, which is equal to the maximum annual dose permitted for a radiation worker according to the recommendation of International Commission on Radiological Protection ${ }^{21}$. 2) It is easy to obtain the material from a human body. That is, the peripheral blood of as little as $5 \mathrm{ml}$ is sufficient. 3) Radiation induced chromosome aberrations can be distinguished from those induced by chemicals if chromosomes are analyzed in the first cell division after exposure, the former being chromosome type while the latter being chromatid type. 4) The rate of spontaneous aberrations, that is, the background frequency of dicentrics and rings is very low.

Disadvantages can be summarized as follows. 1) Only well trained technicians can perform 
the work. Making good chromosome preparations consistently requires expert skills. 2) It is time consuming work. For example, when a whole human body is exposed to $200 \mathrm{KV} \mathrm{X}$-ray at the dose of $250 \mathrm{mGy}$, the frequency of dicentric chromosome is one out of about one hundred metaphase cells. Therefore, thousands of metaphases need to be analyzed per person in a low dose exposure. Thus automatizing the scoring system of radiation induced chromosome aberrations becomes mandatory.

Uniformity and good quality are essentials in preparing the chromosome slide for the automated analysis of radiation induced chromosome aberratioins. In the conventional 2 day blood culture, either whole blood or the enriched layer of white blood cells produced by the gravity is used. Therefore, the number of lymphocytes contained in a specimen is unknown. The cultuer has a large quantity of red blood cells, which are later burst, and the resultant debris remain. Cells other than red blood cells often stick to the bottom of culture flask and are likely to form a cluster. In changing the fixative solutions, cells stick to the wall of a centrifuqe tube or of a pipette, and a great volume of cell is lost. As a result, only experienced workers can save the sufficient number of the lymphocytes without clumping, and can adjust the cell suspension to the optimum concentration for air drying.

In the prsent paper we describe the followings, 1) Lymphocyte culture is far superior to whole blood culture in the study of biological dosimetry. 2) Designing a process for culturing and harvesting lymphocytes in the first cell division towards automatization. 3) The makeup of the robot system constructed based on such a plan. 4) The merits of this robot system demonstrated in its test operation.

\section{MATERIALS AND METHODS}

\section{Biological study.}

The peripheral blood of $11 \mathrm{ml}$ each was drawn from 2 healthy women and a man. The blood was irradiated at the dose of 1 or $3 \mathrm{~Gy}$ (X-rays, $20 \mathrm{~mA}, 200 \mathrm{kV}, 65-70$ rads per minute). Immediately after the irradiation the blood was kept at $37^{\circ} \mathrm{C}$ for 3 hours. In case of nonirradiation the blood was used just after being drawn or after being kept at $37^{\circ} \mathrm{C}$ for 3 hours. To compare whole blood culture with separated lymphocyte culture, one $\mathrm{ml}$ of the blood was taken out from each syringe for the former, and the rest in the respective syringe was used for the latter. The experiments were done five times with irradiated blood. For separating lymphocytes, $10 \mathrm{ml}$ of the whole blood was put into a LeukoPREP tube (Becton Dickinson Co. Ltd) and centrifugalized for 15 minutes at 3,300 r.p.m. (about $1,800 \mathrm{~g}$ ) at $20^{\circ} \mathrm{C}$. Mononuclear cell layer, which usually contains lymphocytes (80\%) and monocytes (20\%), was taken out and mixed into Hanks' balanced salt solution supplemented with $2 \%$ fetal calf serum, and centrifugalized again for 15 minutes at 1,400 r.p.m. (about $330 \mathrm{~g}$ ) at $5^{\circ} \mathrm{C}$. The sediments were resuspended in $5 \mathrm{ml}$ RPMI 1640 medium with $20 \%$ fetal calf serum. The number of cells was then counted with an automatic blood cell counter (Sysmex, Toa Medical Electronics Co., Kobe, Japan). One $\mathrm{ml}$ of the peripheral blood for the whole blood culture and 5 million cells for the separated lymphocyte culture were respectively put into $10 \mathrm{ml}$ culture which consisted of RPMI 
1640 medium, $20 \%$ fetal calf serum and $0.2 \mathrm{ml}$ of PHA (Wellcome Foundation, Ltd.). The cultures were kept in $5 \% \mathrm{Co}_{2}$ incubator at $37^{\circ} \mathrm{C}$. Colcemide $(0.05 \mathrm{micro} \mathrm{g} / \mathrm{ml})$ was given 24 to 3 hours prior to the end of the culture. Cultured cells were collected by the centrifuge, and treated with $0.075 \mathrm{M} \mathrm{KCl}$ hypotonic solution for 20 minutes at $37^{\circ} \mathrm{C}$. They were then fixed with $1: 3$ acetic alcohol. Air-dry slides were made under the warm $\left(29-32^{\circ} \mathrm{C}\right)$ and humid $(70-80 \%)$ condition. For scoring the ratios of the metaphase, simple nucleus, polymorphonucleus and others, we counted the objects found along the vertical line and the horizontal line passing through the center of slide glass under the microscopic view at the magnification of four hundred times.

Examination of the loss of cells by the difference of tools

The loss of cells in a culture flask was examined. Three million mononuclear cells suspended in $6 \mathrm{ml}$ culture medium were put either into a $15 \mathrm{ml}$ polycarbonate (PC) centrifuge tube or into a conventional disposable PC culture flask, respectively. After 2 day culture, the $15 \mathrm{ml}$ tube was centrifuged directly, while the content of the flask was put into a $12 \mathrm{ml} \mathrm{PC}$ centrifuge tube and then centrifuged. The sediments were treated with $2 \mathrm{ml} \mathrm{KCl}$ hypotonic solution in the $15 \mathrm{ml}$ tube or in the $12 \mathrm{ml}$ tube, respectively. Then these specimens were put into $2 \mathrm{ml}$ polypropylene (PP) centrifuge tubes, and washed 3 times with fixative solution according to the standard harvesting method. To keep the cells from being lost by static electricity PP pipette tips were always used in handling the specimens. Final sediments of both samples were diluted in $0.3 \mathrm{ml}$ fixative solution, and 5 micro litter each was placed on a slide glass. The cell density of these two kinds of specimens was compared under the microscope.

Designing a process for culturing and harvesting lymphocytes in the first cell division towards automatization

The trials of the process for culturing and harvesing lymphocytes were performed over a hundred times using the blood of healthy adults and of cancer patients who had been irradiated. The processes were modified many times and the tools for harvest were changed during the trials towards the automatization of preparation system. The final process designed for the automated system is shown in Results section.

\section{Culture and Harvest Robot System (CHROSY)}

Equipments described below were built into the Culture and Harvest Robot System (CHROSY) with or without modification. $\mathrm{CO}_{2}$ gas incubator (CPD-110A, Hirasawa Works Co., Tokyo). Rotator (SR-10N, Ikeda Scientific Co., Ltd., Tokyo). Centrifuge (5100, Kubota Co., Tokyo). Compressor (SC-62, Hitachi Koki Co., Ltd., Ibaragi). Air pump (APN-085V-1, Iwaki Co., Tokyo). Water circulator (CH-201, SCINICS Co., Tokyo). Metal-sensor (OMRON Co., Kyoto). Touch-sensor (OMRON Co., Kyoto). Photo-sensor (Matsushita Electronic Industrial Co., Ltd., Tokyo). Syringe (Hamilton Gas Tight Syringe, Hamilton Co., U.S.A.). $\quad \mathrm{CO}_{2}$ gas cylinder $\left(150 \mathrm{~m}^{3}\right)$. Computer (PC 9801DX, NEC, Tokyo). CRT monitor (PC KD844n, NEC, Tokyo).

The devices manufactured by Umetani Precision Corporation for the present system are as 
follows: Cylinder robot (LaboMaster Jr.); motor manipulated syringe; air manipulated syringe; automatic sliding door; position adjuster; pipette tip rack; capping unit; aluminum racks; servoamplifier; I/O control box; teaching box; pipette tip remover; and cabinet.

\section{Test of CHROSY}

The $\mathrm{CO}_{2}$ incubator being new, some chemical vapor resulting from paintings, coatings, or from the materials for seals affected culture condition. Therefore we could test harvesting process only. The culture samples for trial were cultured for 4 days without $5 \% \mathrm{CO}_{2}$ gas. The culture was started with 0.3 million cells in $6 \mathrm{ml}$ culture medium. Colcemide was given for only one hour. The control experiment of manual harvesting was done at the same time. Samples processed by CHROSY were air-dryed and observed under the microscope.

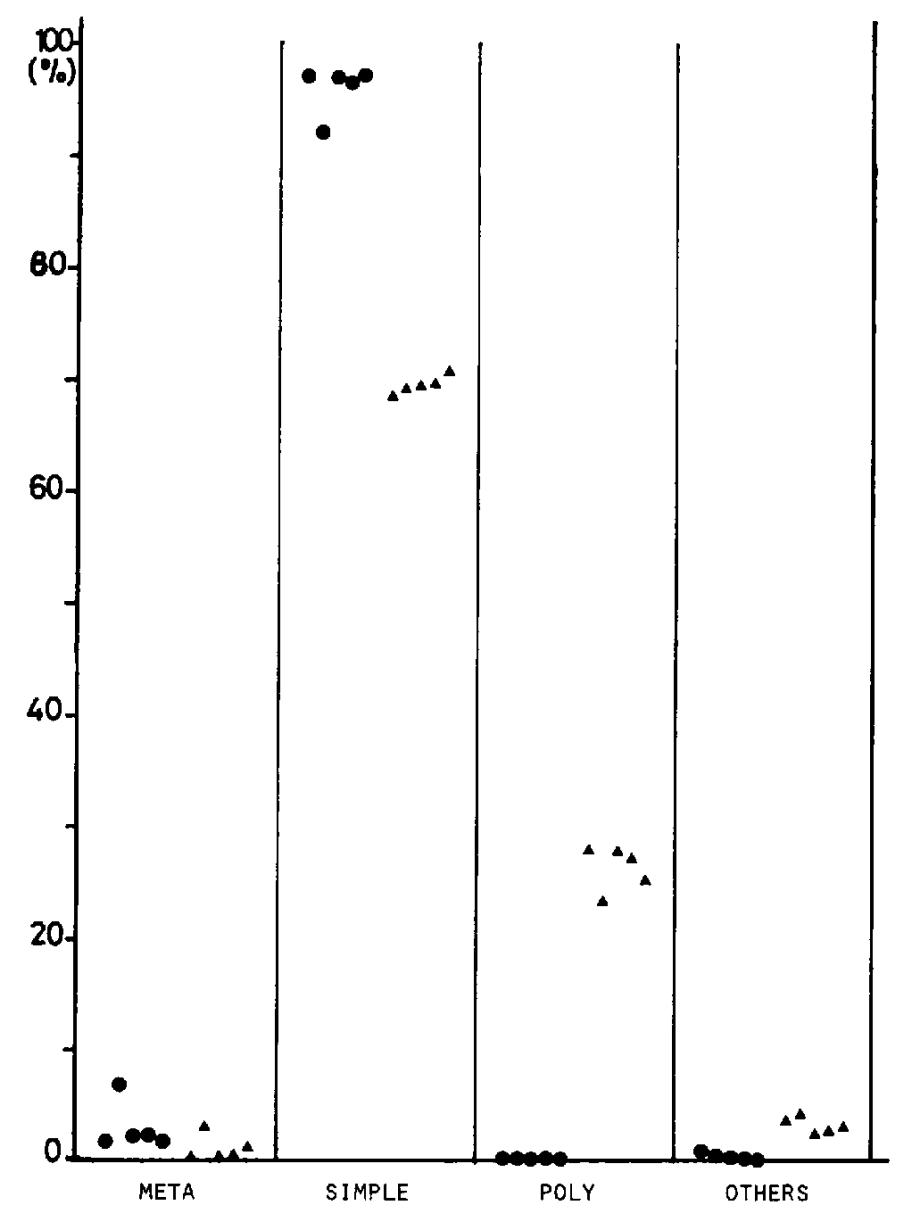

Fig. 1. Difference of frequencies of metaphases, simple nuclei (mainly lymphocytes), polymorphonuclei (granulocytes), and others between 2 day culture of lymphocyte ( $)$ and that of whole blood $(\boldsymbol{\Delta})$. 


\section{RESULTS}

Evaluation of the quality of culture.

For evaluating whole blood culture and lymphocyte culture, we compared the quality of chromosome slides made from those two cultures ${ }^{3,4)}$. The criteria for examining the quality were the frequencies of metaphase, simple nucleus (mainly lymphocyte which has potential to divide responding to PHA), polymorphonucleus, and of other elements including cell debris. Fig. 1 summarizes the result. Five solid circles indicate the results from five cultures with separated lymphocytes, while five solid triangles indicate the results from five cultures with the whole blood. Each circle of five lymphocyte cultures and each triangle of five whole blood cultures correspond with each other from left to right. Frequency of the metaphase was about two times higher in the lymphocyte culture than that in the whole blood culture. The polymorphonucleus was less than $0.5 \%$ in the former and about $25 \%$ in the latter. The rate of unnecessary background objects in the latter was about six times more than that in the former. As shown in Fig. 2 also, it was apparent that the preparation made by lymphocyte culture was superior to that by whole blood culture.

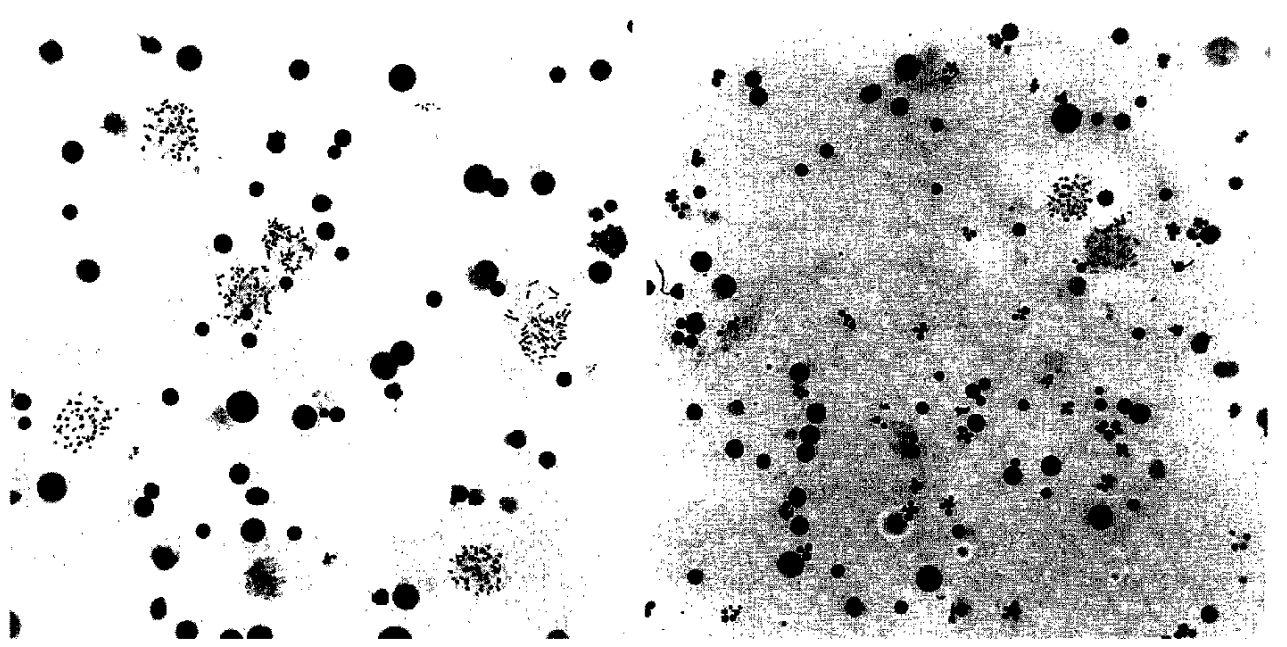

Fig. 2. Air-dry slides made with lymphocyte culture (left) and with whole blood culture (right).

Comparison of the loss of cells caused by the difference of tools.

As shown in Fig. 3 it is obvious that more cells were lost when cultured in the conventional PC culture flask. If glass pipettes and glass centrifuge tubes or $12-15 \mathrm{ml}$ centrifuge tubes had been used during the fixation process, more cells would have been lost.

The process designed towards automatization based on the results mentioned above. Culture

1. Set up a $6 \mathrm{ml}$ culture containing 3 million mononuclear cells in a $15 \mathrm{ml}$ polycarbonate 


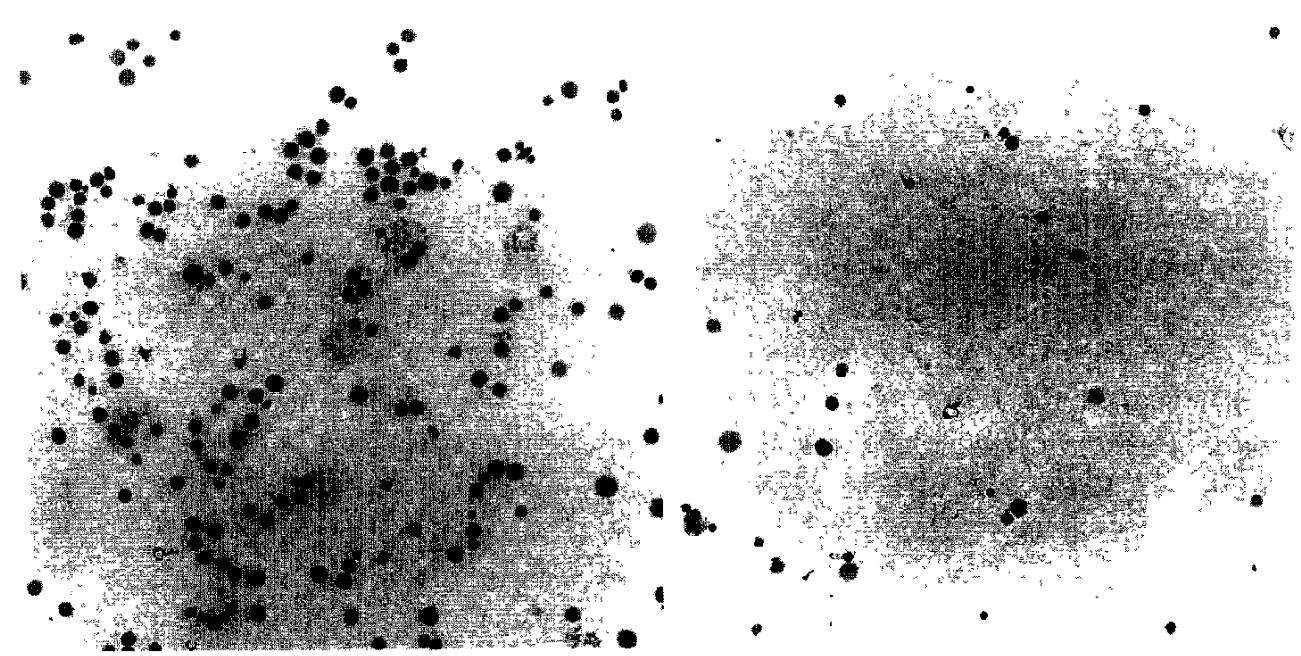

Fig. 3. Air-dry slides made with the samples cultured in the polycarbonate centrifuge tube (left) and in the conventional polycarbonate culture flask (right).

centrifuge tube.

2. Put the tube into the rotator in the incubator and keep it in $5 \% \mathrm{CO}_{2}$ gas for 24 hours at $37^{\circ} \mathrm{C}$.

3. Add colcemide ( 0.3 micro $\mathrm{g}$ ) solution of $0.1 \mathrm{ml}$ into the tube.

4. Continue the incubation for another 25 hours.

\section{Harvest}

5. Centrifugalize the tube at 1,200 r.p.m. (about $240 \mathrm{~g}$ ) for 4 minutes. Remove the supernatant.

6. Mix the sediment well with the $0.075 \mathrm{M} \mathrm{KCl}$ hypotonic solution of $1.5 \mathrm{ml}$ by pipetting and keep it at $37^{\circ} \mathrm{C}$ for 20 minutes.

7. Add the fixative solution (1:3 acetic alcohol) of $0.05 \mathrm{ml}$ into the tube and transfer the content of the tube into the $2 \mathrm{ml}$ small polypropylene centrifuge tube.

8. Centrifuge it at 2,000 r.p.m. (about $310 \mathrm{~g}$ ) for 4 minutes.

9. Remove the supernatant.

10. Add the fixative solution of $1 \mathrm{ml}$ and mix it well by pipetting.

11. Centrifuge it at 2,000 r.p.m. for 4 minutes.

12. Repeat 9 and 10 .

13. Add $1 \mathrm{ml}$ fixative solution and put a cap on it.

14. Store at $4^{\circ} \mathrm{C}$ until the time of air-drying.

This process made it possible to set the concentration of samples simply by adding a fixed volume of solution at the time of air drying. In addition, the sample prepared by this new method offers a) the sufficient number of the final sediment of lymphocyte, b) a chromosome 
slide with a high mitotic index, c) well spread metaphase, and d) a clear background. We can obtain more than 20 chromosome slides from one culture tube.

\section{Culture and Harvest Robot System (CHROSY)}

For incorporating the design mentioned above into a system, a robot for culturing and harvesting lymphocyte chromosomes was constructed. The makeup is shown in Figs. 4 and 5 . Equipments on the platform in the cabinet of CHROSY are arranged clockwise surrounding a central handling robot as follows: A rotator incubator with an automatic sliding door; a pipette tip remover; a centrifuge equipped with a position adjuster and an automatic sliding door; a cool $\left(4^{\circ} \mathrm{C}\right)$ aluminum block bath \& a warm $\left(37^{\circ} \mathrm{C}\right)$ aluminum block bath; two pipetting and aspirator nozzles connected to air pumps; a pipette tip rack; and a capping unit with a nozzle for $\mathrm{CO}_{2}$ gas. A compressor, an air pump for the aspirators, an air manipulated syringe, $\mathrm{I} / \mathrm{O}$ control box and two water circulators are placed under the platform. A personal computer with CRT, a servoamplifier and a teaching box are placed on an ordinary desk. The desk and a $\mathrm{CO}_{2}$ gas cylinder are placed next to the cabinet. A bottle or a centrifuge tube is carried by the mandible equipped at the end of the robot hand. A pipetter ( $5 \mathrm{ml}$ syringe) is also placed at the terminal

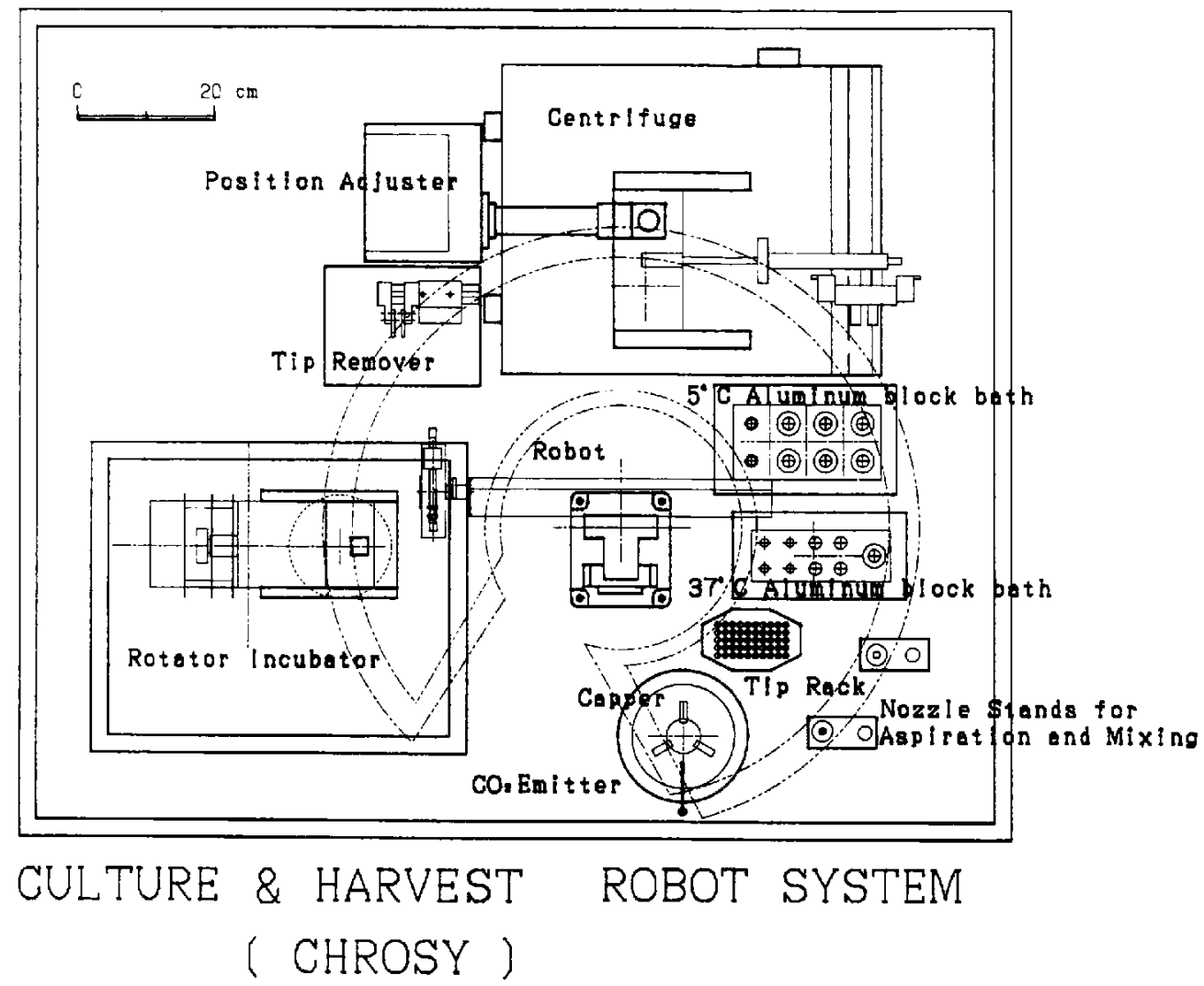

Fig. 4. A schematic figure of the main part of Culture and Harvest Robot System (CHROSY). 


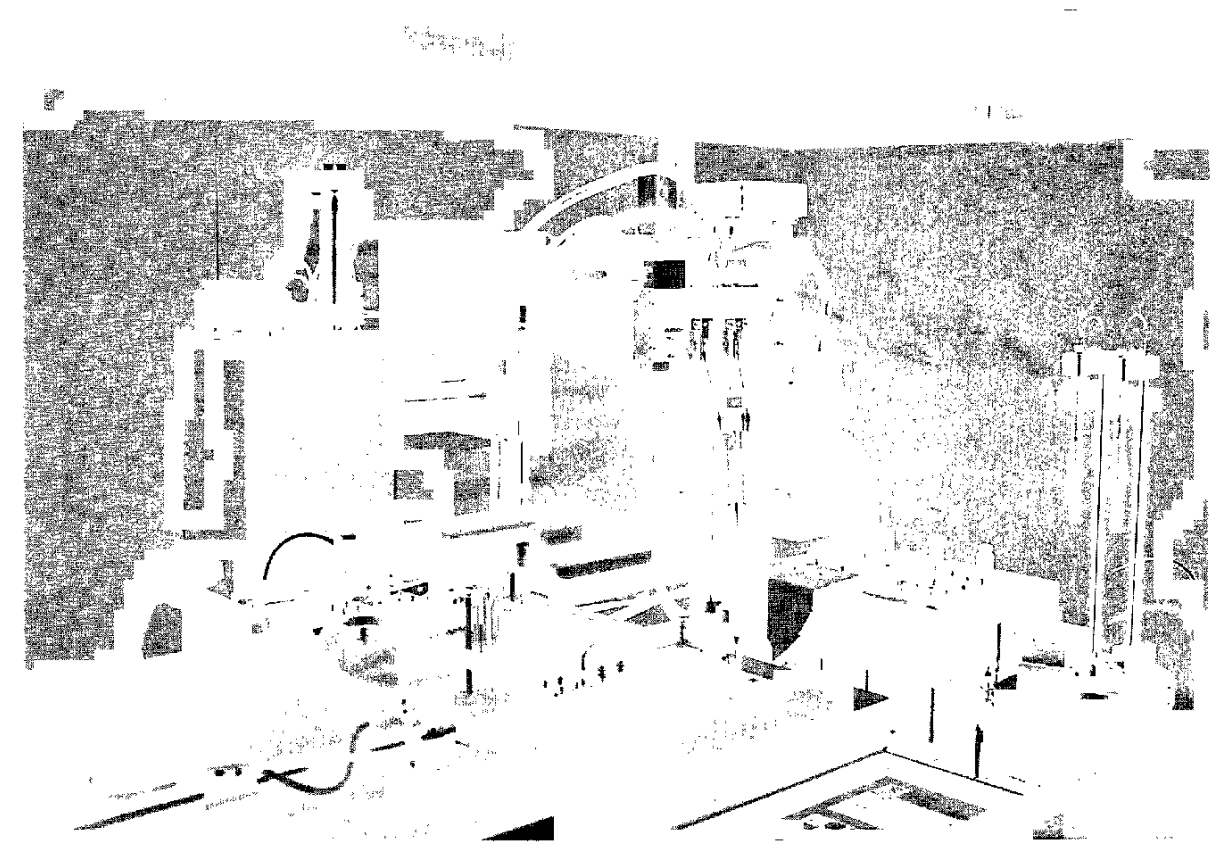

Fig. 5. A photograph showing the main part of CHROSY. A mandible of central handling robot holds a centrifuge tube. A pipetter is lying above the mandible.

Table 1. Time for processing 2 samples.

\begin{tabular}{lcc}
\hline Work & CHROSY & Manual \\
\hline Add cells and PHA & $20 \mathrm{~min}$ & $4 \mathrm{~min}$ \\
Add colcemide & $12 \mathrm{~min}$ & $2 \mathrm{~min}$ \\
Harvest & $2 \mathrm{~h} 25 \mathrm{~min}$ & $1 \mathrm{~h} 4 \mathrm{~min}$ \\
\hline
\end{tabular}

Table 2. Adjustable parameters in CHROSY's program

\begin{tabular}{lr}
\hline Volume of sample & (to total $6.0 \mathrm{ml})$ \\
Volume of PHA & $(0.1-1.0 \mathrm{ml})$ \\
Time for first incubation & $(0.2-96.0 \mathrm{hr})$ \\
Volume of colcemide & $(0.1-1.0 \mathrm{ml})$ \\
Time for second incubation & $(0.2-96.0 \mathrm{hr})$ \\
Rising speed of robot hand & $(0.07-1.4 \mathrm{~mm} / \mathrm{s})$ \\
Volume of KCl solution & $(0.1-8.0 \mathrm{ml})$ \\
Volume of 1 drop fixative & $(0.05-1.0 \mathrm{ml})$ \\
Volume of fixative & $(0.1-8.0 \mathrm{ml})$ \\
Mixing time by pipetting & $(1-9 \mathrm{times})$ \\
\hline
\end{tabular}


of the hand. It can draw the solution of $0.05-1.5 \mathrm{ml}$ at a time (the maximum limit depends on the capacity of the pipette tip used). The working limit of the robot is as follows: Vertical, 30 $\mathrm{cm}$; horizontal, $42 \mathrm{~cm}$; rotation, 300 degrees; wrist twisting, 300 degrees; and mandible opening, $6 \mathrm{~cm}$. Positioning accuracy is within $0.1 \mathrm{~mm}$.

The control system program is written in Basic and Assembly languages on MS-DOS. The program can be changed by different parameter input so that the robot can work regardless of the differences in the volume of solution, incubation time, and in mixing time by pipetting (Table 2). Since the robot can move just like a human hand, it is possible to design many procedures for a variety of culturing and harvesting methods using CHROSY.

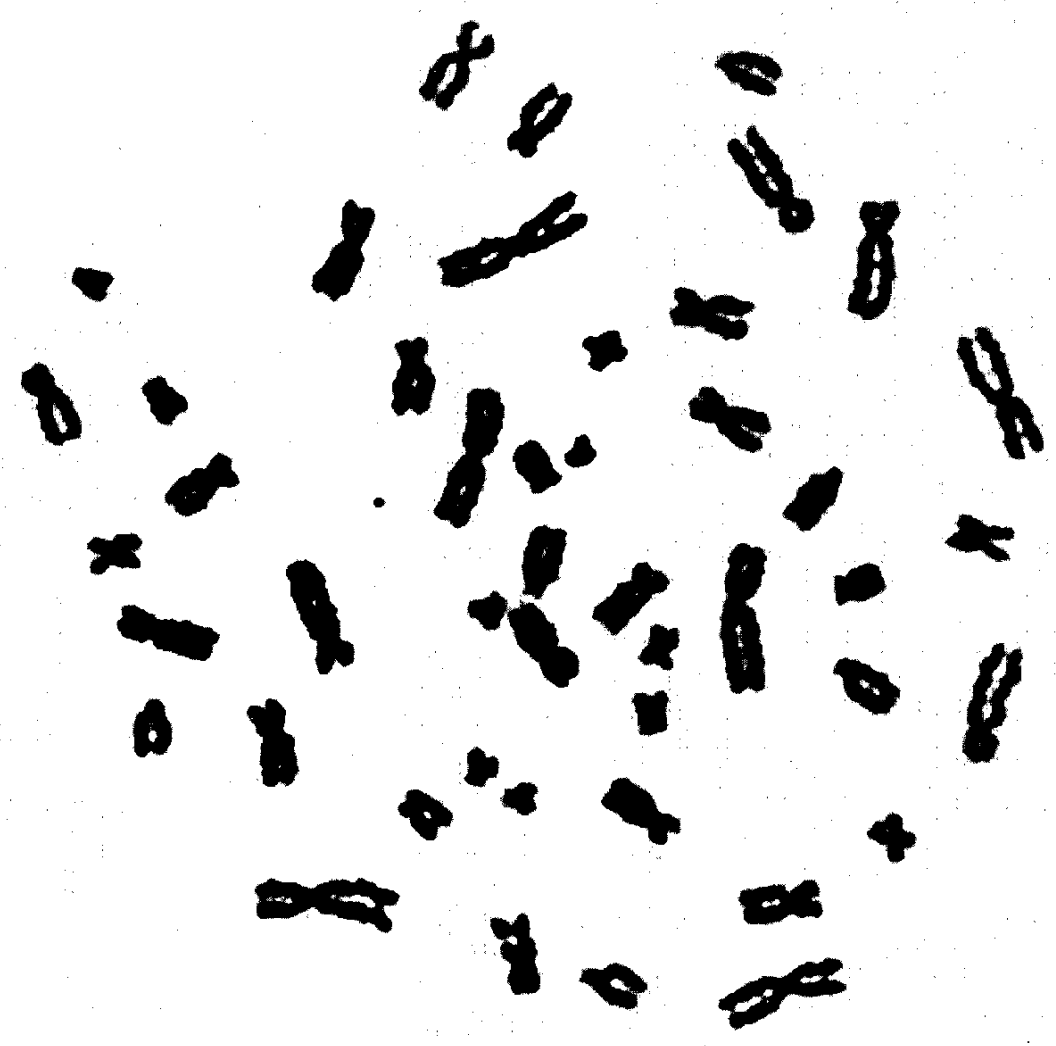

Fig. 6. A metaphase observed on the air-dry slide made with the sample CHROSY harvested. 


\section{Test of performance}

In the present operation we could obtain such results as shown in Table 2. Although the spreading of metaphases was better in the air-dry slides made from the sample harvested manually, it was possible to make a fairly good chromosome slide from the sample harvested by CHROSY automatically. One example of the metaphases treated by CHROSY is shown in Fig. 6.

\section{DISCUSSION}

There are only few studies on the automatization of the culture and harvest for chromosome preparation. An automated equipment for the fluid aspiration and dispensing is helpful for chromosome preparation ${ }^{5,6)}$. The harvesting steps are semiautomatically performed by TECAN (TECAN, U.S. Ltd., Chapel Hill, U.S.A. and Hombrechtikon, Switzerland; GI-RO 3283 Multislide Machine, MA. re, Milan, Italy; CROMAT 90, S \& H Milan, Italy; Etaleur Chaffant Type Ect 85, Productions John Toulemonde \& Cie, Paris, France; Machine Lab Tech, Sermeter, Niles, IL, U.S.A.) $)^{7)}$.

Full-scale automated systems for chromosomal preparation were developed at the City of Hope National Medical Center and the Jet Propulsion Laboratory of California Institute of Technology, California ${ }^{8,9)}$, and at the University of Leiden, Netherlands ${ }^{10)}$. The former was designed to process a large number of peripheral blood specimens at a time. This system is capable of making air-dry slides also. On the other hand, the latter was constructed to perform the simultaneous processing of small sets of samples according to various culturing methods. Different from these systems, the purpose of CHROSY is, even if it takes more time than manual work does, to produce a good and uniformed chromosome preparation for air-drying. The good and uitiformed slides prepared automatically will no doubt offer a breakthrough in the quality of biological preparations for the automated analysis and heighten the reliability of the analysis because they guarantee the sure data constantly. It will eliminate such a conventional problem that the figures to be analyzed vary widely depending on technicians who prepare the specimens. In our test operation even a complete beginner could operate CHROSY meaning the skipping of the years of trainings. Connecting good quality with high speed is always an ideal goal. We will eventually set our target at increasing CHROSY's capacity of a day.

At present we are in the process of testing the performance of CHROSY. As demonstrated in the results it was possible to obtain fairly good sample for air-drying by CHROSY. The test was done with samples cultured without the supplement of $5 \% \mathrm{CO}_{2}$ gas. The result will be better if $5 \% \mathrm{CO}_{2}$ gas is supplied and the $\mathrm{pH}$ of the culture is adjusted properly during the incubation. We hope that this new robot system will make a contribution to setting a new standard of chromosome preparation for automated scoring system of radiation induced chromosome aberrations. 


\section{ACKNOWLEDGEMENTS}

We would like to thank Mr. S. Sekiyama, Umetani Precision Co., for the engineering work and Miss. J. Matsuoka for the assistance in the biological experiment. This work was supported by the project grant for the study of Assessment \& Reduction of Radiation Risks from the Science and Technology Agency, Japan.

\section{REFERENCES}

1. Takahashi, E., Hirai, M., Tobari, T., Utsugi, T. and Nakai, S. (1982) Radiation-induced chromosome aberration in lymphocytes from man and crab-eating monky. The dose-response relationships at low doses. Mut. Res. 84: 115-123.

2. International Commission on Radiological Protection (1977) Recommendation of the International Commission on Radiation Protection, ICRP Publication 26, Pergamon Press.

3. Hayata, I. and Yamamoto, K. (1989) Modification of the chromosomal preparation for the automated analysis. The 11th European Workshop on Automated Cytogenetics. Besse en Chandesse, 14-17 September.

4. Hayata, I., Minamihisamatsu, M., Sato, K., Muto, M. and Yamamoto, M. (1990) Improved chromosome preparation for radiation dosimetry. Annual Report (1989-1990) of the National Institute of Radiological Sciences. pp. 42.

5. Wulf, H. C. (1975) Mechanical preparation of cells for chromosome studies. Hum. Hered. 25: 398-401.

6. Spureck, J., Carlson, R., Allen, J. and Dewald, G. (1988) Culturing and robotic harvesting of bone marrow, lymph nodes peripheral bloods, fibroblasts, solid tumors with in situ techniques. Cancer Genet. Cytogenet. 32: 59-66.

7. Martin, A. O., Shaunnessey, M., Sabrin, H., Maremont, S., Dyer, A., Cimino, M. C., Rissman, A., McKinney, R. D., Cohen, M., Jenkins, E. C., Kowal, D. and Simpson, J. L. (1989) Evaluation and development of a system for automated preparation of blood specimens for cytogenetic analysis. In "Automation of Cytogenetics", Ed. C. Lundsteen and J. Piper, pp. 149-173, Springer-Verlag, Berlin, Heidelberg, New York.

8. Melnyk, J. H., Persinger, G., Mount, B. and Castleman, K. R. (1970) A semi-automated specimen preparation system for cytogenetics. J. Reprod. Med. 17: 59-67.

9. Castleman, K. R. and Melnyk, J. H. (1975) Final Report. NIH No1-HD-5-2843.

10. Vrolijk, J., Korthof, G., Vletter, G., van der Geest, C. R. G., Gerrese, G. W. and Pearson, P. L. (1989) An automated system for the culturing and harvesting of human chromosome specimens. In "Automation of Cytogenetics", Ed. C. Lundsteen and J. Piper, pp. 135-148, Springer-Verlag, Berlin, Heidelberg, New York. 